\title{
Collective Flow Measurements from the PHENIX Experiment
}

\author{
ShinIchi Esumi (for the PHENIX Collaboration \\ Inst. of Physics, Univ. of Tsukuba, \\ Tennno-dai 1-1-1, Tsukuba, Ibaraki 305-8571, Japan \\ E-mail: esumi@sakura.cc.tsukuba.ac.jp
}

\begin{abstract}
Recent collective flow measurements including higher moment event anisotropy from the PHENIX experiment are presented, and the particle type, beam energy dependence and the relation with jet modification are discussed. The measured higher order event anisotropy with event plane defined at forward rapidities and the long range correlation with large $\eta$ gaps are both consistent with initial geometrical fluctuation of the participating nuclei. In $200 \mathrm{GeV} \mathrm{Au}+\mathrm{Au}$ collisions, higher order event anisotropy, especially simultaneous description of $\mathrm{v}_{2}$ and $\mathrm{v}_{3}$, is found to give an additional constraining power on initial geometrical condition and viscosity in the hydrodynamic calculations. $\mathrm{v}_{2}, \mathrm{v}_{3}$ and $\mathrm{v}_{4}$ are almost unchanged down to the lower colliding energy at $39 \mathrm{GeV}$ in $\mathrm{Au}+\mathrm{Au}$. The measured two particle correlation with subtraction of the measured $\mathrm{v}_{n}$ parameters shows a significant effect on the shape and yield in the associate particle $\Delta \phi$ distribution with respect to the azimuthal direction of trigger particles. However some medium responses from jet suppression or jet modification seems to be observed. Direct photon $\mathrm{v}_{2}$ has been measured in $200 \mathrm{GeV}$ $\mathrm{Au}+\mathrm{Au}$ collisions. The measured $\mathrm{v}_{2}$ is found to be small at high $\mathrm{p}_{\mathrm{T}}$ as expected from non-suppressed direct photon $\mathrm{R}_{\mathrm{AA}} \simeq 1$, which can be understood as being dominated by prompt photons from initial hard scattering. On the other hand, at lower $\mathrm{p}_{\mathrm{T}}<4$ $\mathrm{GeV} / \mathrm{c}$ it is found to be significantly larger than zero, which is comparable to other hadron $\mathrm{v}_{2}$, where thermal photons are observed.
\end{abstract}

\section{Higher order event anisotropy measurements}

In order to measure the higher order event anisotropy, the event planes from the higher moments at various rapidities are defined with various reaction plane detectors; the Reaction Plane detector (RXN: $|\eta|=1.0-2.8$ ), the Muon Piston Calorimeter (MPC: $|\eta|=3.1$ - 3.7), the Beam-Beam Counter (BBC: $|\eta|=3.1$ - 3.9) and the Zero Degree Calorimeter (ZDC: $|\eta|>6.5$ ). Fig,1 shows the event plane correlations between forward and backward rapidities for the same moments in panels (a) and (b), for the different moments in panels (c) and (d). The significant positive correlations between the same order higher moments are visible in panel (b), which is consistent with the initial geometrical fluctuation[1]. A weak negative correlation between $1^{\text {st }}$ and $3^{\text {rd }}$ moment

$\ddagger$ A list of members of the PHENIX Collaboration can be found at the end of this issue. 



Figure 1. higher order event plane correlation between same and different harmonics and between different rapidities, where the subscript $n=1 \sim 4$ and superscript $\mathrm{X}=\mathrm{A} \sim \mathrm{F}$ indicated on $\Phi_{\mathrm{n}}^{\mathrm{X}}$ in the figure are the order of harmonics and the index of the following sub-detectors, respectively; A:RXN(+) ${ }^{(+)} \mathrm{B}: \mathrm{BBC}^{(-)}, \mathrm{C}: \mathrm{MPC}^{(+)}, \mathrm{D}: \mathrm{MPC}^{(-)}, \mathrm{E}: \mathrm{BBC}^{(+)}$ and $\mathrm{F}: \mathrm{ZDC}^{(+/-)}$, where $(+)$or $(-)$indicates the positive or the negative rapidity. The descriptions of the sub-detectors and their $\eta$ acceptances are given in the text.

event planes can be an indication of rapidity anti-symmetric $\mathrm{v}_{3}$ contribution as seen in panel $(\mathrm{d})$, which is further confirmed by the correlation measurements with respect to the $1^{\text {st }}$ moment event plane from the spectators shown in panels (e) and (f), where the weak anti-symmetric $\mathrm{v}_{3}$ contribution shows the same sign as $\mathrm{v}_{1}$ at the same rapidity. No visible correlation between $2^{\text {nd }}$ and $3^{\text {rd }}$ harmonics seen in panel $(\mathrm{d})$, this also indicates that the presence of geometrical fluctuation is the dominant effect.

The measured $\mathrm{v}_{2}, \mathrm{v}_{3}$ and $\mathrm{v}_{4}$ with respect to the same order event plane $\Psi_{n}$ defined at forward rapidity in RXN are shown as a function of $\mathrm{p}_{\mathrm{T}}$ for several centrality slices in each panel in Fig, 2 2] and as a function of $\mathrm{N}_{\text {part }}$ for two $\mathrm{p}_{\mathrm{T}}$ bins in Fig. 2] 2], $\mathrm{N}_{\text {part }}$ dependence is compared with a generalized eccentricity up to the higher moments. Significant higher moments compared to $\mathrm{v}_{2}$ are observed for central collisions, and smaller centrality dependence is seen especially in $\mathrm{v}_{3}$. One should also note that this $\mathrm{v}_{4}\left(\Psi_{4}\right)$ seems to be larger than the previously measured $\mathrm{v}_{4}\left(\Psi_{2}\right)$ [3], because of the additional fluctuation contribution in $\Psi_{4}$ angle with respect to $\Psi_{2}$ angle.

The measured $\mathrm{v}_{2}$ and $\mathrm{v}_{3}$ are compared with various model calculations in Fig, 4, Since the initial generalized eccentricities $\epsilon_{n}$ between Glauber model and CGC-KLN model have opposite trends for $2^{\text {nd }}$ and $3^{\text {rd }}$ moments because of different fluctuation contribution with respect to the initial overlapping geometry, the shear viscosity over entropy ratio $\eta / s$ used in hydrodynamic calculations will be more constrained by 


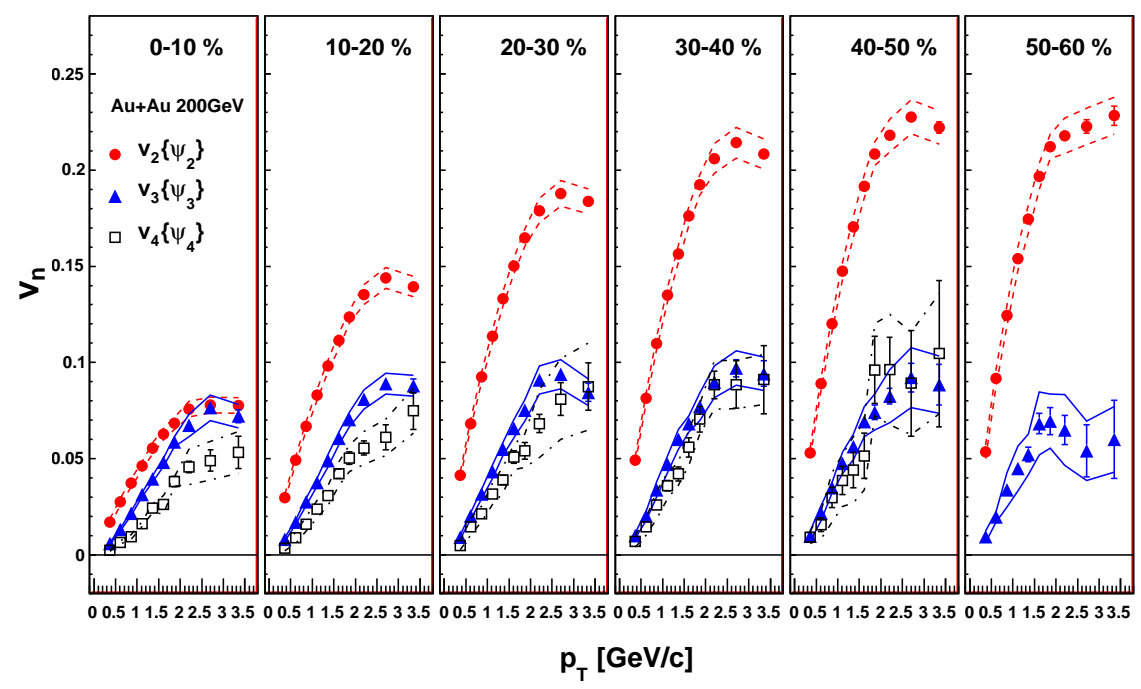

Figure 2. measured $\mathrm{v}_{n}$ parameters as a function of $\mathrm{p}_{\mathrm{T}}$ for different centrality slices at $200 \mathrm{GeV} \mathrm{Au}+\mathrm{Au}$ collisions

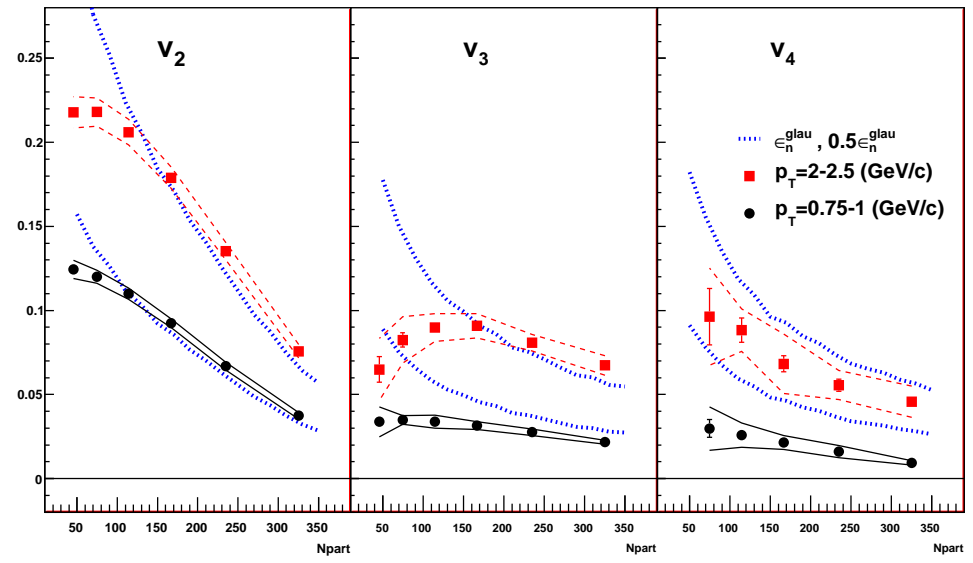

Figure 3. measured $\mathrm{v}_{n}$ parameters as a function of $\mathrm{N}_{\text {part }}$ (for two different $\mathrm{p}_{\mathrm{T}}$ bins) at $200 \mathrm{GeV} \mathrm{Au+Au} \mathrm{collisions}$

simultaneous fitting to the measured $\mathrm{v}_{2}$ and $\mathrm{v}_{3}$, compared with the previous $\mathrm{v}_{2}$ alone fitting. The existing hydrodynamic calculations seem to describe the experimental measurements using the Glauber type initial condition with a smaller shear viscosity and the other calculations with the MC-KLN type initial condition with a larger shear viscosity are disfavored by the data [4, 5].

Identified hadron $\mathrm{v}_{3}$ is shown as a function of $\mathrm{p}_{\mathrm{T}}$ for $\pi^{+-}, K^{+-}$and $p+\bar{p}$ in Fig.5. The similar mass ordering of $\mathrm{v}_{3}$ compared with $\mathrm{v}_{2}$ is observed at relatively lower $\mathrm{p}_{\mathrm{T}}$ region as one might expect from the radial flow picture from hydrodynamic expansion, while at intermediate $\mathrm{p}_{\mathrm{T}}$ region, the similar baryon - meson splitting in $\mathrm{v}_{3}$ given by 


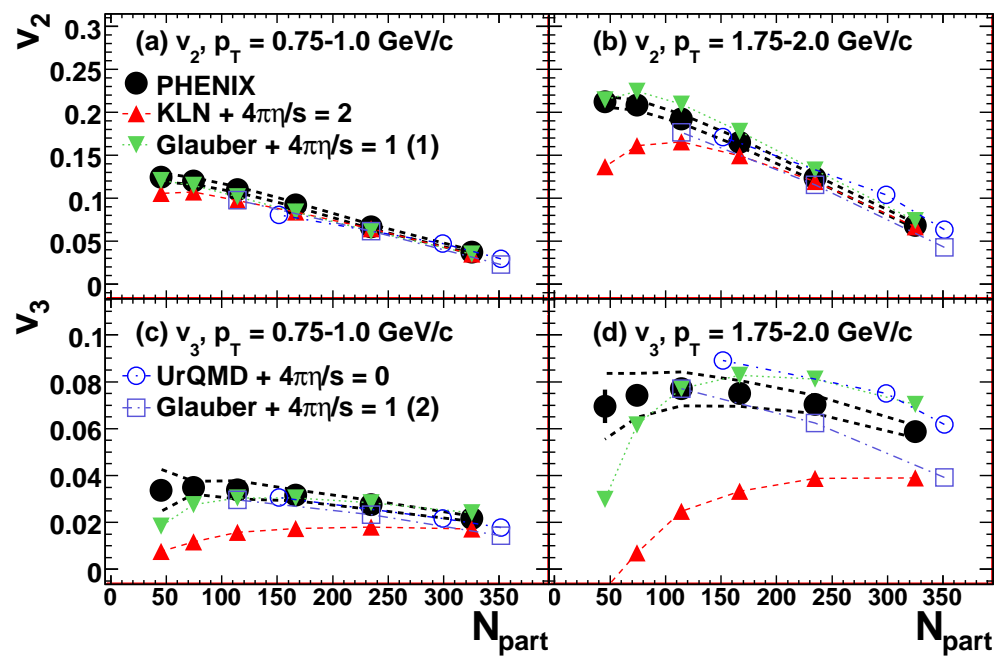

Figure 4. comparison of $\mathrm{v}_{2}$ and $\mathrm{v}_{3}$ with various model calculations

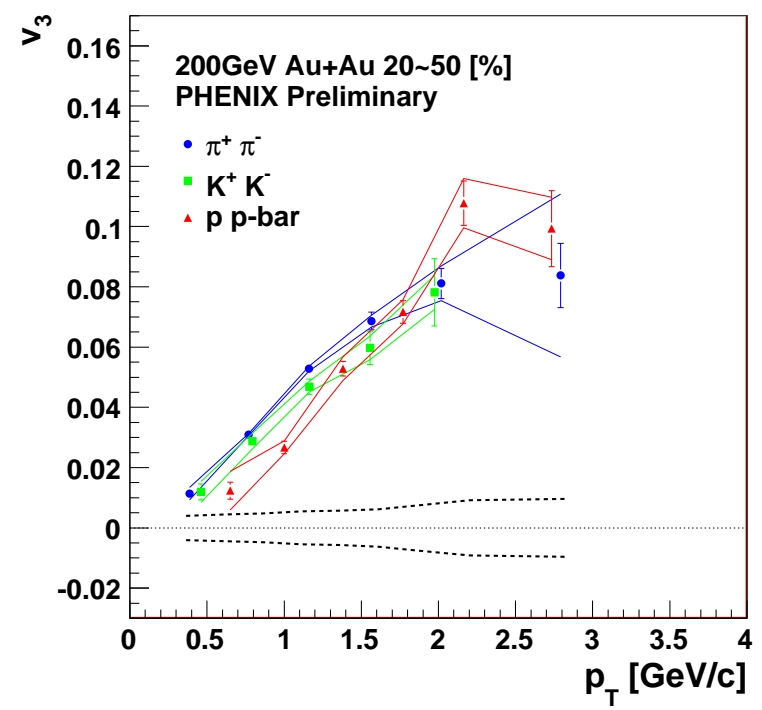

Figure 5. Identified hadron $\mathrm{v}_{3}$ as a function of $\mathrm{p}_{\mathrm{T}}$ at $200 \mathrm{GeV} \mathrm{Au}+\mathrm{Au}$ collisions

the number of constituent quarks could possibly be seen just like the quark number scaling of $\mathrm{v}_{2}$, which might be related to the partonic degrees of freedom during the $\mathrm{v}_{3}$ evolution. Both observations at least tell us that the $v_{3}$, which is originated from the initial geometrical fluctuation, seems to reflect the hydrodynamic evolution as triangular expansion.

The higher harmonics event anisotropy $\mathrm{v}_{n}$ measurements for charged particles and the identified hadron $\mathrm{v}_{2}$ measurements has also been done for both $62 \mathrm{GeV}$ and 39 $\mathrm{GeV} \mathrm{Au}+\mathrm{Au}$ collisions, the observed $\mathrm{v}_{n}$ and $\mathrm{v}_{2}$ results for given $\mathrm{p}_{\mathrm{T}}$ and centrality show very similar results to those of $200 \mathrm{GeV} \mathrm{Au}+\mathrm{Au}$ collisions, which would indicate that 
the hydrodynamical properties are mostly unchanged within the collision energy region 39-200 GeV[6].

\section{Implication on two-particle correlation measurements}

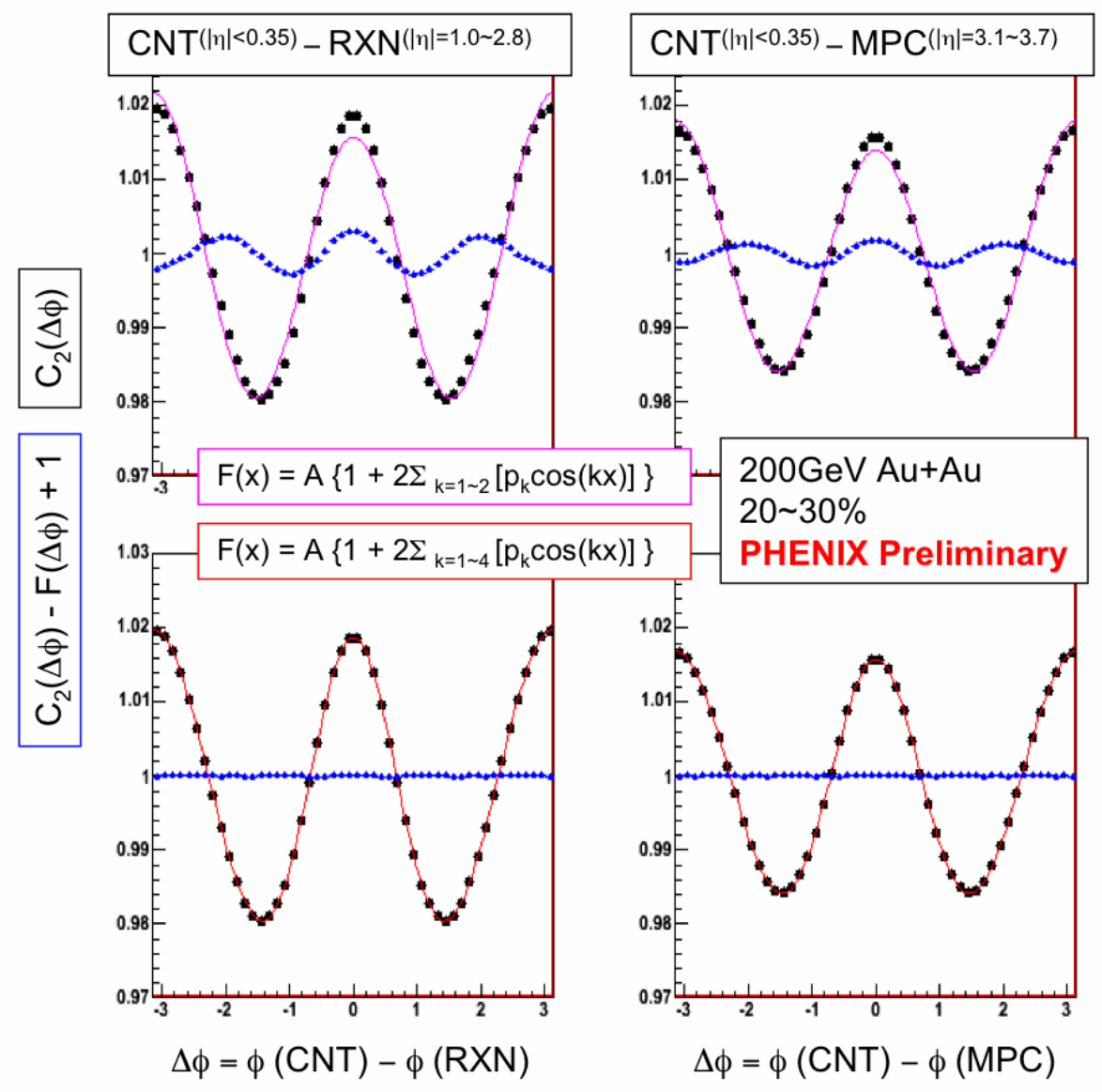

Figure 6. two particle azimuthal correlation between forward and central rapidities

Fig, [6 shows the two particle azimuthal correlation between forward and central rapidities, where the left panels are with RXN and the right panels are with MPC for forward rapidity. The both top and bottom panels show the same data points (black) and the data are fitted with the Fourier function shown as red curves up to the $2^{\text {nd }}$ order for top panels and up to the $4^{\text {th }}$ order for the bottom panels. The difference between data points and fitted curve is shown as blue points, where the correlation is well described by including up to the $4^{\text {th }}$ order as shown in the bottom panels, while the differences shown in top panels show significant $3^{\text {rd }}$ moment signal, which has previously been considered as the near-side ridge and away-side double-peak like structure. These fitted parameters can then be used to extract the higher order event anisotropy and has been confirmed to be consistent with the results from the event plane method. One has to keep in 
mind that the same experimental observations (near- and away-side structures) are just expressed in a different way by the higher order harmonic parameters $\mathrm{v}_{n}$.

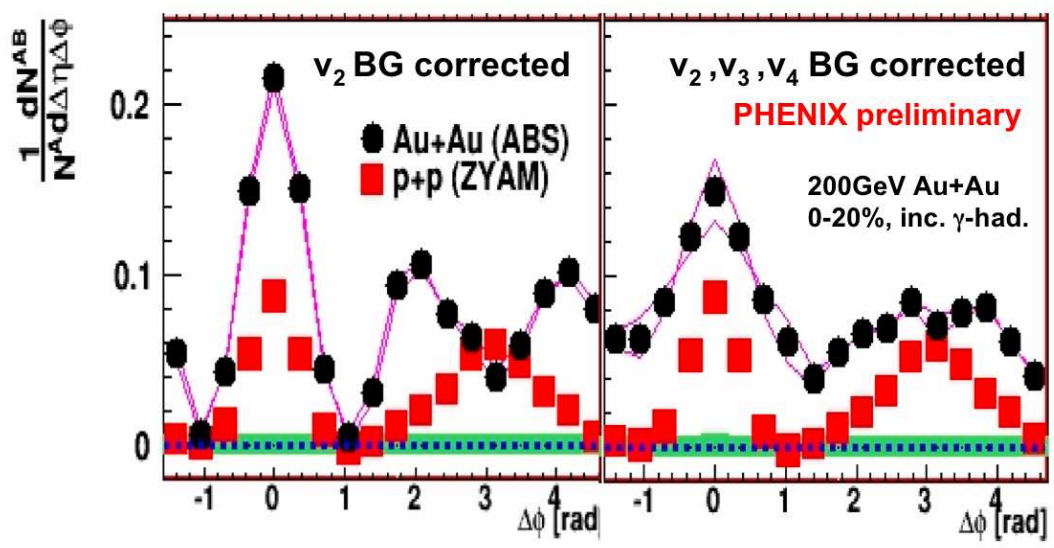

Figure 7. two particle azimuthal correlation within the central rapidities

Fig.7 shows the inclusive $\gamma$-hadron correlation measured within the central rapidity region $(|\eta|<0.35)$ in central $\mathrm{Au}+\mathrm{Au}$ collisions compared to $\mathrm{p}+\mathrm{p}$ collisions [7]. The left panel shows the result with the $\mathrm{v}_{2}$ BG correction, while the right panel shows the corrected results with the measured $\mathrm{v}_{2}, \mathrm{v}_{3}$ and $\mathrm{v}_{4}$ parameters. The jet associated yields for $\mathrm{Au}+\mathrm{Au}$ collisions are determined by the absolute normalization method without the ZYAM assumption. Although the away-side double-peak like structure has been significantly reduced with including the higher moment parameters $\mathrm{v}_{n}$ in the BG correction, there seems to be some remaining effects from the jet suppression and/or modification in terms of broadening and enhancement in both near- and away-side shape relative to the shape in $\mathrm{p}+\mathrm{p}$ collisions.

\section{Direct photon elliptic event anisotropy measurements}

The direct photon carries various different contributions; (1) prompt photon from initial hard scattering at high $\mathrm{p}_{\mathrm{T}}$, which is expected to give zero $\mathrm{v}_{2},(2)$ fragmentation photon after the parton energy loss, which should follow the hadron as positive $v_{2}$, (3) jet conversion photon and Bremstrahlung photon, which would have negative $\mathrm{v}_{2}$, since this is a part of energy loss itself, (4) thermal photon, which would carry the history of the dynamical evolution of quark gluon plasma. The direct photon $\mathrm{v}_{2}$ has been determined by the measured $\pi^{0}$ and inclusive photon $\mathrm{v}_{2}$ and relative direct photon yield over the total inclusive photon yield including various hadronic decay photons. This measurement has been extremely difficult because of the relatively small direct photon signal especially at low $\mathrm{p}_{\mathrm{T}}$ region. Using the virtual photon measurement [8, 9], the accuracy of the relative direct photon yield determination over the total inclusive photon yield has been greatly improved. This improvement has given a significant reduction of systematic error on the extracted direct photon $\mathrm{v}_{2}$ value below $4 \mathrm{GeV} / \mathrm{c}$ in $\mathrm{p}_{\mathrm{T}}$ in the following figures. The 

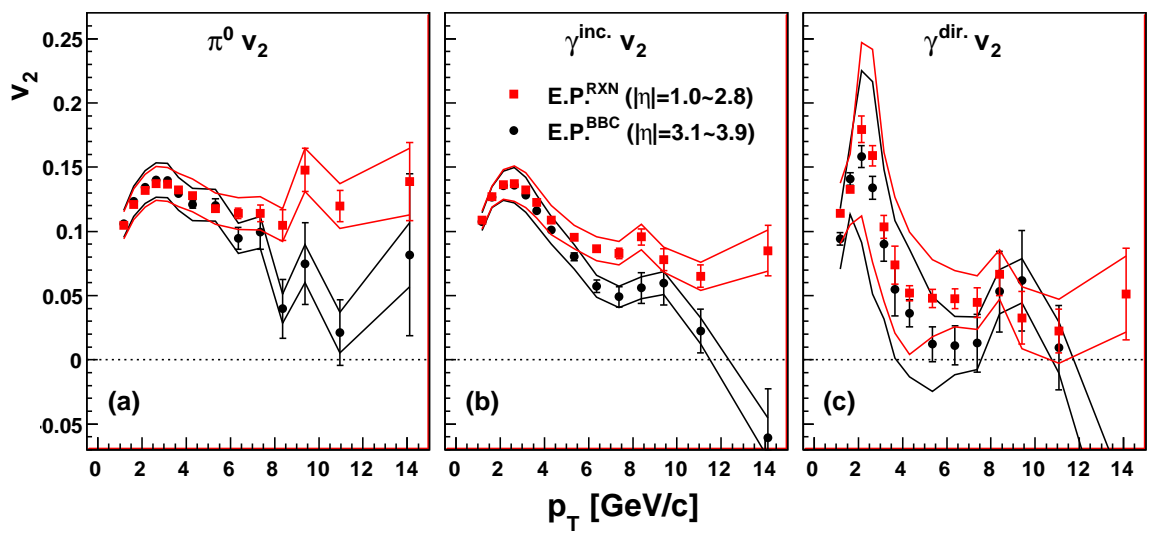

Figure 8. $\pi^{0}, \gamma^{\text {inc. }}$ and $\gamma^{\text {dir. }} \mathrm{v}_{2}$ parameters as a function of $\mathrm{p}_{\mathrm{T}}$ at $200 \mathrm{GeV} \mathrm{Au}+\mathrm{Au}$ minimum bias collisions
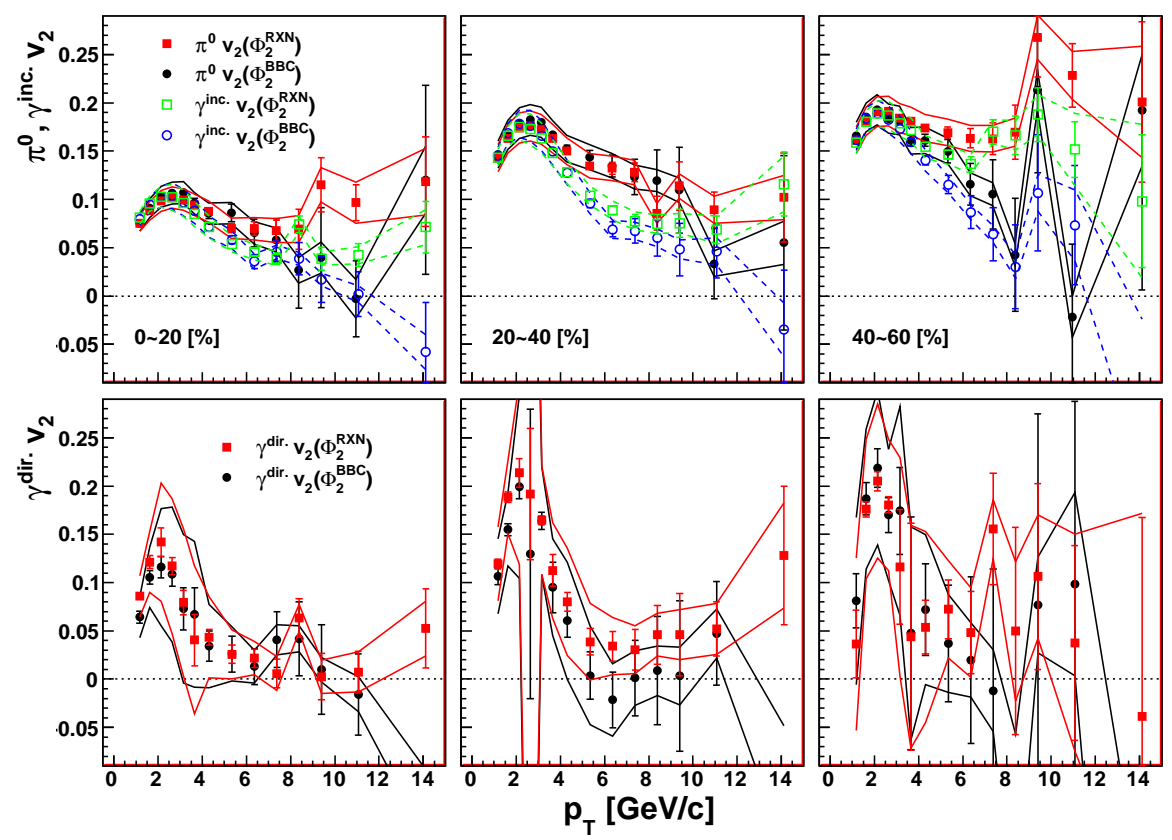

Figure 9. $\pi^{0}, \gamma^{\text {inc. }}$ and $\gamma^{\text {dir. }} \mathrm{v}_{2}$ parameters as a function of $\mathrm{p}_{\mathrm{T}}$ for three centrality selections at $200 \mathrm{GeV} \mathrm{Au}+\mathrm{Au}$ collisions

measured $\pi^{0}, \gamma^{\text {inc. }}$ and $\gamma^{\text {dir. }} \mathrm{v}_{2}$ are shown in Fig, 8 and Fig, as a function of $\mathrm{p}_{\mathrm{T}}$ in 200 $\mathrm{GeV} \mathrm{Au}+\mathrm{Au}$ collisions for minimum bias and for three centrality bins, respectively[10]. The data are shown with two event planes defined at two different rapidities. The difference between two event planes is clearly seen for $\pi^{0}$ and $\gamma^{i n c} \mathrm{v}_{2}$ at higher $\mathrm{p}_{\mathrm{T}}$ region expected from the possible non-flow contributions, however the difference in the $\gamma^{\text {dir. }} \mathrm{v}_{2}$ is smaller than the systematic error bands.

At the higher $\mathrm{p}_{\mathrm{T}}$ region, the direct photon $\mathrm{v}_{2}$ is consistent with zero as also seen in Fig 10, which confirms the previous direct photon $\mathrm{R}_{\mathrm{AA}}$ measurement to be about 1 because of the dominant prompt photon production. On the other hand, at the lower 
$\mathrm{p}_{\mathrm{T}}$ region as shown in Fig, 8 and Fig, 9 , the direct photon $\mathrm{v}_{2}$ is found to be significantly larger than zero and similar to the hadron $\mathrm{v}_{2}$. Various model calculations assuming entire history of thermal photon emissions which includes a significant early time (small $\mathrm{v}_{2}$ ) contribution have failed to explain the experimental measurement.

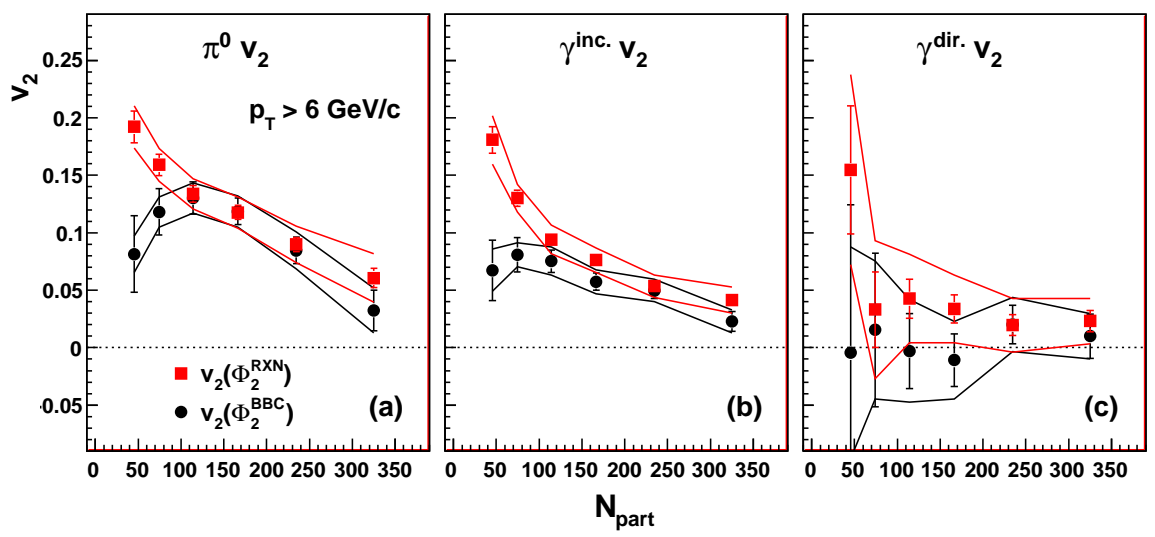

Figure 10. $\pi^{0}, \gamma^{\text {inc. }}$ and $\gamma^{\text {dir. }} \mathrm{v}_{2}$ parameters (integrated above $6 \mathrm{GeV} / \mathrm{c}$ ) as a function of $\mathrm{N}_{\text {part }}$ at $200 \mathrm{GeV} \mathrm{Au}+\mathrm{Au}$ collisions

\section{Conclusion}

The recent results on the collective flow measurements from the PHENIX experiment are presented. The higher order event anisotropy has been measured to be consistent with initial geometrical fluctuation followed by hydrodynamic expansion. The initial condition and shear viscosity in hydrodynamic calculations are found to be more constrained by these measurements. This also strongly influences on the understanding of the extracted shape of two particle correlation from the jet-medium interaction. The direct photon elliptic event anisotropy $\mathrm{v}_{2}$ has been observed to be small as expected from the prompt photon production at high $\mathrm{p}_{\mathrm{T}}$, while it has been observed to be as large as the hadronic $\mathrm{v}_{2}$ at lower $\mathrm{p}_{\mathrm{T}}$, where thermal photons are seen.

\section{References}

[1] B.Alver and G.Roland, Phys. Rev. C81, 054905 (2010).

[2] A.Adare et al., arXiv:1105.3928

[3] A.Adare et al., Phys. Rev. Lett. 105, 062301 (2010).

[4] B.Alver et al., Phys. Rev. C82, 034913 (2010).

[5] B.Shenke et al., Phys. Rev. Lett. 106, 042301 (2011).

[6] X.Gong, R.Lacey et al., This issue of proceedings.

[7] S.Bathe, R.Lacey et al., This issue of proceedings.

[8] A.Adare et al., Phys. Rev. Lett. 104, 132301 (2010).

[9] A.Adare et al., Phys. Rev. C81, 034911 (2010).

[10] A.Adare et al., arXiv:1105.4126 